\title{
7. ON THE ROLE OF DIFFUSE EMISSION NEBULAE IN GALAXIES
}

\author{
The Late G. A. ShaJN \\ Simeis Astrophysical Observatory, Crimea, U.S.S.R.
}

(Read by Mrs Masevich)

The emission nebulae, as more or less compact gaseous or gas-and-dust formations, belong to a rather widespread type of population in our Galaxy. Though, for well-known reasons, we are able to penetrate into the depth of the Galaxy no further than $2-3 \mathrm{kpc}$ for $\mathrm{H}$ in regions, several hundred nebulae have already been recorded (predominantly in the spiral arms). Sometimes the emission nebulae almost entirely occupy large regions of the sky, as, for instance, in the region of $\rho, \gamma$ and $o^{1}$ Cygni. In addition, in the area within latitudes $\pm 5^{\circ}$, we observe a nearly continuous faint emission background [1].

Emission nebulae are undoubtedly widespread in extra-galactic spiral systems also. We were able to investigate in detail with our 64 -cm camera (F I.4) only the nearest spiral systems: $M_{3}$ I, $M_{33}, M_{51}, M_{1}$ Ior, $\mathrm{NGC}_{2403}, \mathrm{NGC}_{3627}, \mathrm{NGC}_{425}$, NGC $463 \mathrm{r}$. According to Baade and Mayall, there are hundreds of emission nebulae in $\mathrm{M}_{3} \mathrm{I}$ located along the spiral arms [2]. Over a hundred emission nebulae were found in $\mathbf{M}_{33}$ with the aid of moderate-size instruments $[3,4,5]$. By using our photographs taken with a red $\mathrm{H} \alpha$ filter, a narrow violet one $[\lambda 3727$ (O II)] and an ordinary yellow one, we were able to obtain the distribution of the

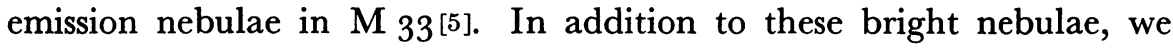
suggested the presence of a considerable number of fainter nebulae, and also of many patches of an emission character. In the other spiral galaxies mentioned above, we also found a number of bright emission nebulae. The majority of these were known or had been suggested earlier $[6,7,8]$. The faintest nebulae recognized with certainty by us are about $15^{\mathrm{m} \cdot 7}$ (red magnitude). This corresponds approximately to the absolute magnitude $-6^{\mathrm{m}} \cdot \mathrm{o}$ for $\mathrm{M}_{33}$ and $\mathrm{M}_{3} \mathrm{I}$ and to $-7^{\mathrm{m}} \cdot 7^{7}$ for the other galaxies mentioned above (or $\mathrm{I}^{\mathrm{m}} \cdot 5$ brighter according to Baade's new distance scale). The integral absolute magnitude of the Orion nebula, NGC ${ }_{1976}$, is about $-6^{\mathrm{m}} \cdot 5$ at the same wave-length. Therefore, the faintest emission nebulae 
recorded by us are no fainter intrinsically than NGC 1976 ; the majority of them are brighter or much brighter than the brightest nebulae in the accessible part of the sky.

The above-mentioned spiral galaxies were selected only because they are the nearest to us. Therefore, there is no reason to consider them exceptional, particularly in respect to their gaseous population. There is no doubt that in each of these galaxies, in addition to the brighter emission nebulae which we have recorded, there are hundreds of other nebulae as in $\mathbf{M}_{33}$ and $\mathbf{M}_{3} \mathrm{I}$. The galaxies under consideration are all of the $\mathrm{Sc}$ and $\mathrm{Sb}$ types. It seems that $\mathrm{M}_{3} \mathrm{I}$ is no poorer in emission nebulae than our Galaxy, while other galaxies are no poorer than $\mathrm{M}_{3} \mathrm{I}$ and may be even richer. For example, the emission nebulae in $\mathrm{M}_{33}$ are brighter than those in $\mathrm{M}_{3} \mathrm{I}$ (by $\mathrm{I}^{\mathrm{m}} \cdot \mathrm{O}$ or $\mathrm{I}^{\mathrm{m}} \cdot 5$ in the mean). Therefore, the nearest galaxies selected for a more detailed study are at least as rich in their gaseous population as is our Galaxy; galaxies like $\mathrm{M}_{33}$ and $\mathrm{M}$ Ior are much richer.

On the other hand, there are reasons to believe that the spiral galaxies under discussion do not differ at all from other spiral galaxies. This holds true particularly for the Sc galaxies. One can hardly doubt that emission nebulae are present in practically all galaxies. If these galaxies could be studied in detail, we should find them nearly as rich in nebulae as $\mathbf{M}_{3} \mathrm{I}$, $\mathrm{M}_{33}, \mathrm{M}_{5} \mathrm{I}, \mathrm{M}$ IoI, NGC 2403, etc. For this reason, one can assume that richness in nebulae distributed along the spiral arms is a characteristic feature of $\mathrm{Sb}$ and $\mathrm{Sc}$ galaxies. The problem of emission in the central part is not so clear.

The peculiarities of the spiral galaxies discussed above are of great interest in connexion with the question of the relative age of the galaxies as a whole and of the emission nebulae contained in them. Usually $3 \times 10^{9}$ years is assumed as a lower limit for the age of galaxies in the stage we are now observing. On the other hand, there are important reasons to believe that the age of the diffuse emission nebulae, as more or less compact formations, does not exceed $10^{7}$ years, i.e. that it is about a hundred times less than the age of the galaxy as a whole.

Some arguments in favour of the short age of emission nebulae are given below.

(a) The observed structure of several emission nebulae in the form of rings or arcs may be interpreted as an indication of the outward motion of the matter and, therefore, of a recent origin of these nebulae as well as of their dissipation in the near future [9].

(b) The existence of groups of emission nebulae for which there are serious reasons for believing that the total energy is positive [9]. 
(c) The expected expansion of the emission nebulae and their dissipation as a result of temperature and pressure differences between the nebulae and the surrounding cold regions. Even the strong magnetic field $\left(\sim \mathrm{IO}^{-4}\right.$ gauss) in the inner part of the Orion nebula does not prevent it from expansion and dissipation [9].

(d) The disintegration of emission nebulae because of collisions (once in $10^{7}$ years on the average). This argument was suggested by Oort for interstellar clouds in general[10].

The result depends to a large extent on whether or not the nebula and its exciting star are connected genetically. There are important reasons to think that the connexion is genetic, but even if it is only temporary and accidental, the nebula has enough time to expand and dissipate before getting out of the ionization zone of the exciting star. It is easy to see that doubling the size of a nebula leads to a decrease in the emission measure by a much greater factor (thirty-two times).

These arguments lead to the conclusion that the age of the diffuse nebulae in the emission stage is really short, of the order of $10^{7}$ years or less, i.e. about a hundred times less than the age of the galaxies as a whole.

This result must be considered reliable. It is also necessary to consider as certain, the result that all spiral galaxies, or at least the overwhelming majority of them, are very rich in emission nebulae and that the latter are located along the spiral arms to a great extent. There are two possible explanations:

(I) We observe our Galaxy in a peculiar stage when these short-lived objects are formed. But emission nebulae occur in all spiral galaxies. This means that if this hypothesis is true it is necessary to assume that such a peculiar stage has set in for all galaxies at the same time. Such a supposition seems quite improbable.

(2) Emission nebulae originate more or less continuously, after which they disintegrate, dissipate, or are possibly transformed into other objects. There are some reasons to conclude that nebulae originate in groups. Some important consequences of this hypothesis must be emphasized now. From the age of the diffuse nebulae ( $\sim 10^{7}$ years or less) and their mean spatial velocity of $10 \mathrm{~km} / \mathrm{sec}$ or more, we find that the nebulae have been formed not far from their present locations in the spiral arms, probably within several hundred parsecs. But we observe nebulae along the entire spiral arms. Therefore, we must assume that the nebulae have originated and continue to originate almost everywhere along the spiral arms. Evidently, we must also conclude that the spiral arms themselves are 
very steady formations, notwithstanding the short age of their constituents (gas, dust, and hot stars) [9].

If the age of emission nebulae is shorter than the age of the galaxies by at least a hundred times and the nebulae themselves disintegrate and dissipate within this period (less than $10^{7}$ years) it is to be expected that a great quantity of matter, at least a hundred times as much as is now present in the form of emission nebulae, has dissipated during the age of the Galaxy $\left(\sim 3 \times 10^{9}\right.$ years $)$. We cannot estimate the total mass of nebulae, but if it is of the order of a few per cent of the total mass of the stars, the total mass of the material scattered during $3 \times 10^{9}$ years would be more than the total mass of all the stars.

If the dissipation is a one-sided process, we should arrive at a contradiction with the well-known upper limit for the mass of diffuse matter derived by Oort.

At present we can observe only the process of disintegration and dissipation of the emission nebulae, but it seems probable that some other kind of transformation of the diffuse matter in the form of ions, neutral atoms and dust also exists, a process which must be intimately related to the formation of stars.

In the problem under consideration, the possible effect of the interstellar magnetic field deserves attention. It is known that the magnetic lines of force seem to be oriented along the spiral arms. In addition, we found recently that the magnetic field is probably responsible for the greatly elongated shape of many emission and dark nebulae. Therefore, it may be sufficiently effective to control the distribution and motion of diffuse matter in the Galaxy to some extent[11]. If so, the magnetic field will probably contribute to the conservation of the diffuse matter as a system and prevent it from scattering freely, notwithstanding the above-mentioned dissipation of the emission nebulae. In any case, the scattering will probably be restricted in the presence of a sufficiently strong interstellar magnetic field. This means that the magnetic field will contribute to the conservation of the spiral structure of galaxies, at least their gas and dust population. However, the emission nebulae and the hot stars are so intimately related that the last suggestion probably applies to the O-B stars also-another important constituent of the population of the spiral arms.

\section{REFERENGES}

[1] Shajn, G. A. and Hase, V. F. Atlas of Diffuse Gaseous Nebulae, 1952.

[2] Baade, W. and Mayall, N. U. Probl. of Cosm. Aerodyn. 1951, p. 165.

[3] Haro, G. A.J. 55, 66, $195^{\circ}$. 
[4] Aller, L. H. Manuscript, Michigan, I95I.

[5] Shajn, G. A. Comptes rendus de l'Acad. d. Sci. de l'U.R.S.S. 93, no. 6, 993, 1953.

[6] Mayall, N. U. and Aller, L. H. Publ. A.S.P. 51, I 12, 1939.

[7] Seyfert, C. W. $A p . J .91,528$, r 940.

[8] Courtès, G. C. R. 234, 506, 1952.

[9] Shajn, G. A. and Hase, V. F. Comptes rendus de l'Acad. d. Sci. de l'U.R.S.S. 96, no. 5, 945, 1954; no. 6, I 129, 1954; Publ. Crim. Obs. 8, 8o, 1952.

[10] Oort, J. H. Probl. of Cosm. Aerodyn. 1951.

[I I] Shajn, G. A. A.J. U.S.S.R. 32, I 10, 1955. 